\title{
Colloidal suspensions in confined geometries
}

\author{
Clemens Bechinger* \\ University of Konstanz, Physics Department, Fach M676, D-78457 Konstanz, Germany
}

\begin{abstract}
In the presence of geometric confinements such as topographically patterned surfaces, strong light fields, or channels the phase behavior and the dynamical properties of colloidal suspensions are strongly modified in comparison to the bulk properties. Because such geometric confinements play an important role in many physical, biological and chemical processes, investigations of the properties of colloidal suspensions in such situations may help to obtain a better understanding of, e.g. transport of particles through cell membranes or catalytic reactions in zeolitic materials. (c) 2002 Elsevier Science Ltd. All rights reserved.
\end{abstract}

Keywords: Colloidal suspensions; Two-dimensional phase transitions; Patterned substrates; Entropic forces; Optical tweezers; Single-file diffusion

\section{Introduction}

Colloidal suspensions, i.e. well-defined mesoscopic particles which are suspended in a fluid, have received increasing attention amongst scientists. Because colloidal particles rapidly sample their accessible configurational space, they can be regarded as analog computers which allow real time 'simulations' and therefore substantially contribute to the understanding of processes in atomic systems such as nucleation and growth phenomena [1], melting and freezing [2-4], glass transition [5], etc. In contrast to atomic systems, where the pair interaction is fixed by the electronic structure of the corresponding atoms, the interaction between colloids can be tailored over a large range. In addition to very short-range steric interactions (similar to ideal hard spheres) also charge stabilized colloids with long-range repulsion are available and thus allow to adopt the particle interaction appropriate to very specific situations. Accordingly, colloidal suspen-

\footnotetext{
* Tel.: +49-7531-883562; fax: +49-7531-883127.

E-mail address: Clemens.Bechinger@uni-konstanz.de (C. Bechinger).
}

sions have been established as versatile model systems for physicists, chemists and biologists.

The intention of this review is to highlight some recent experimental and theoretical work on colloidal suspensions where the emphasis is not on bulk effects, but on phenomena which are induced or at least strongly pronounced by the presence of geometrical constraints such as patterned walls, pores or strong light fields. It will be demonstrated that such confinements lead to strong deviations compared to bulk systems with respect to the phase behavior, but also affects the diffusion behavior of colloidal particles. Aside from fundamental interest, the study of confined geometries is also motivated by the ongoing rapid development in the field of nano-technology where surfaces and confinements become increasingly important.

\section{Colloidal crystallization on patterned substrates}

In a number of experiments hard sphere-like colloidal dispersions have been demonstrated to crystallize on flat, uniform surfaces with a random stacking of closed packed planes [6,7]. This is due to the fact 
that face-centered-cubic (fcc) and hexagonal closed packed (hcp) crystals have almost the same free energy as confirmed by computer simulations [8]. Accordingly, a mixture of both stacking types, i.e. a random stacking of closed packed planes is usually obtained under real conditions. However, when a periodically patterned surface is used as a template, directed crystallization of bulk colloidal crystals can be observed, thus permitting the tailoring of the lattice structure, orientation and size of the resulting crystals. Since such crystals can be used as optical switches and photonic band gap materials [9], the guided growth of such structures is-in addition to fundamental studies of epitaxial growth of thin crystalline layers - also of high technical relevance.

It has been demonstrated by van Blaaderen et al. that if a PMMA layer with holes arranged in a square lattice is used as a substrate, a single-crystal is formed which extends over many lattice spacings perpendicular to the substrate [10]. The depth of the holes was chosen to be close to the radius of the particles and accordingly, the energy gain of a colloidal sphere dropping into a hole corresponded to approximately $0.6 k_{B} T$ which is large enough to stabilize the structure at room temperature.

The stabilization of crystalline structures on patterned substrates can be considerably enhanced when smaller particles or non-adsorbing polymers are added to the colloidal suspension. It is well known that under these conditions, the smaller particles induce entropic forces between the larger colloidal particles and the wall [11-13]. In addition to forces perpendicular to the wall, the presence of patterned substrates [14] or curved surfaces [15] leads to horizontal depletion forces which can be utilized to enhance two-dimensional (2-D) positional order of colloidal particles in the presence of substrate patterns. Lin et al. showed that the combination of entropic forces with patterned substrates leads to a rich variety of 2-D fluid like and solid like phases [16]. Fig. 1a-c are micrographs of a 2-D colloidal system (particle diameter is $1 \mu \mathrm{m})$ on top of three different patterned substrates with a schematic reconstruction in the bottom left corner and the computed structure factor in the top right corner. The parameter $\chi$ denotes the ratio between the particle diameter and the spatial period of the substrate pattern. Because $\chi=0.75$ in Fig. 1a, no coupling of the sterically stabilized colloidal particles across adjacent grooves occurs and a modulated liquid is observed. In contrast, when $\chi=2.02$, as this is the case in Fig. 1b, adjacent lines of colloidal particles are in registration thus forming a 2-D crystalline phase. Fig. 1c shows an example of a 2-D colloidal system on top of a 2-D patterned substrate $(\chi=1)$. As can be seen by the corresponding a confocal image in Fig. 1d, the substrate induces larger fcc crystals. Because the entropic forces increase the energy gain of a particle dropping into a substrate hole
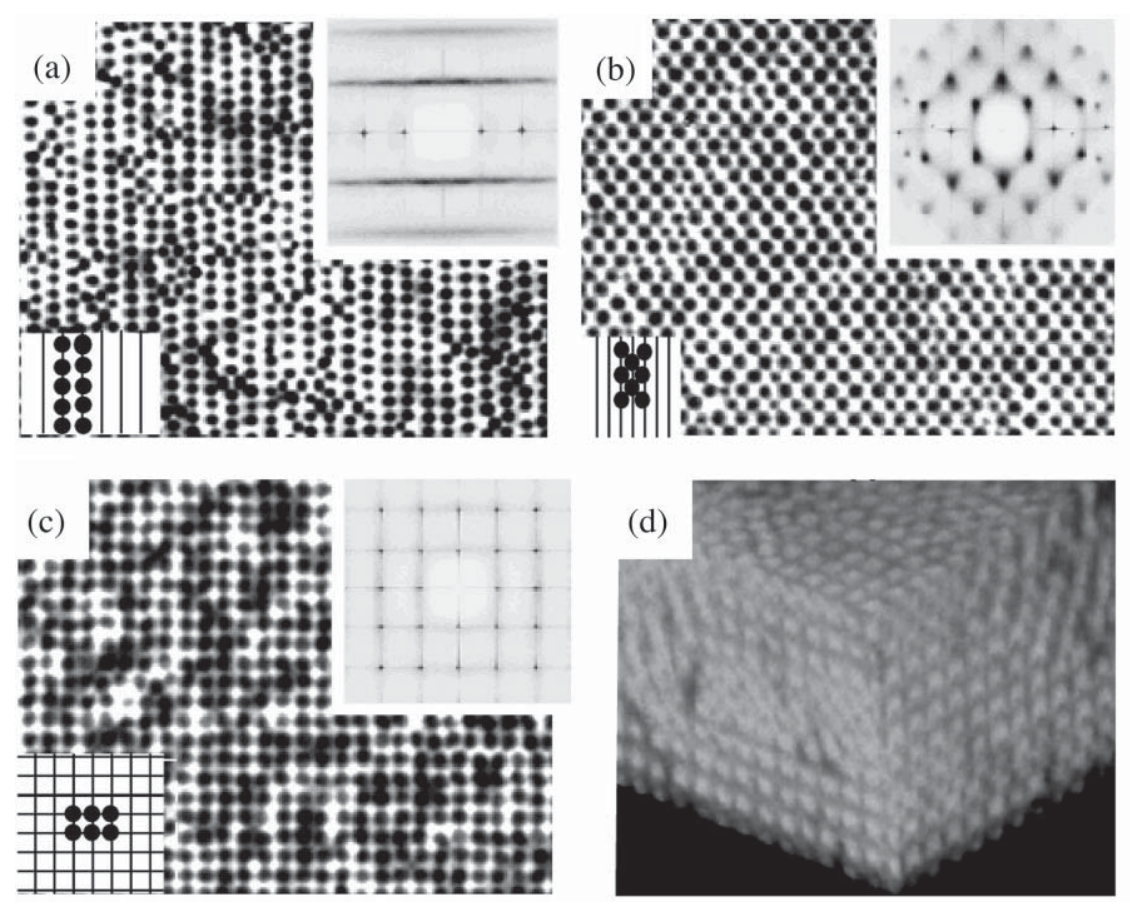

Fig. 1. Micrographs of 2-D entropically driven assembly of colloids on different substrate patterns (a)-(c). The inset corresponds to a schematic reconstruction of the colloidal monolayer on the substrate. (d) is a three-dimensional confocal image showing 20 layers of a larger (>30 layer) FCC crystal grown on a template as in (c), but derived using slightly different particle and polymer concentrations [16]. 
by more than one order of magnitude, the combination of entropic attraction and surface patterns provides a simple and robust route towards controlled colloidal self-assembly in 2-D and 3-D. Entropic forces do not apply to particular length scales, therefore the underlying principle should be also applicable on smaller, e.g. macromolecular dimensions.

\section{Surface freezing of hard sphere fluids on patterned substrates}

The effect of patterned substrates on the crystallization behavior of hard sphere fluids has been also investigated by Heni et al. using computer simulations $[17,18]$. It could be shown that close to a topographically patterned wall the spheres start to crystallize below the corresponding bulk freezing point. The onset of this so-called precrystallization can be significantly shifted away from coexistence by using pattern geometries, which are commensurate with the corresponding bulk crystal. In the case of (111) and (110) patterned substrates, complete wetting of the substrate by a hard sphere fluid is observed where first a crystalline layer close to the surface is formed which is followed by a cascade of layering transitions as the bulk freezing point is approached (Fig. 2). In contrast, incomplete wetting is observed for (100) and (110) substrates. By applying substrate patterns which are somewhat distorted or which have a lattice constant mismatch with respect to the hard sphere crystal, incomplete wetting of the substrate, i.e. only a finite number of 2-D crystalline layers is observed as the bulk co-existence is approached. This number depends significantly on the amount of lattice distortion with respect to an ideal lattice, which is commensurate with a coexisting crystal. However, although no complete wetting is observed in this situation, it is possible to shift the onset of prefreezing to even lower pressures by slightly expanding the wall pattern compared to the ideal-matched co-existing crystal. Furthermore, even a commensurate wall pattern can lead to incomplete wetting as demonstrated for the (100) and (110) pattern. In the case of highly distorted rhombic patterns, precrystallization is completely suppressed.

\section{Colloidal particles in 2-D optical lattices}

Instead of topographically patterned surfaces, substrate potentials can be also obtained by means of strong optical light fields which can be conveniently produced by lasers. Due to the polarizability of colloidal particles, they experience light forces which are directed into the region of highest intensity (gradient

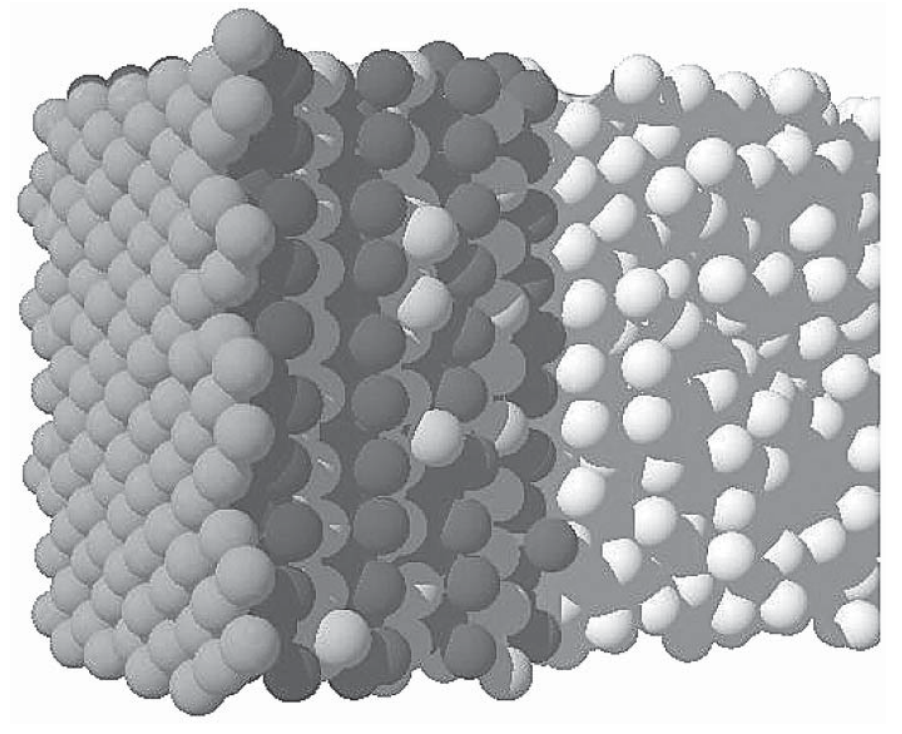

Fig. 2. Configurational snapshot of a hard sphere system on a (111) substrate (the latter is not shown for clarity). The first few layers are in perfect registration with the substrate (left) while at larger distances the system becomes fully fluid (right). To demonstrate the frozen nature of the first few layers they are plotted in different gray scales [18].

forces) $[19,20]$. When several laser beams are overlapped, the corresponding interference pattern provides a convenient and flexible way to produce optical substrate potentials [21,22]. Because the geometry of such a modulated light field can be easily varied by, e.g. changing the angle of incidence of the laser beams, optical substrate potentials are very useful for systematic studies of 2-D systems on patterned substrates. Even more important than the high flexibility regarding the geometry of light-induced substrate potentials, however, is the possibility that the substrate strength $V_{0}$ can varied in situ by changing the laser intensity or the polarization of the laser beams, respectively. It is worth noting that the latter is usually not possible in atomic systems.

Brunner et al. have created 2-D substrate potentials by overlapping three laser beams which form an interference pattern corresponding to a (111) surface [23]. The number density of the colloidal particles was chosen in such a way that each potential well was occupied by three particles, i.e. a trimer, at sufficiently high laser intensities.

The particle density was adjusted by a scanned optical tweezer which acted as a boundary box for the investigated 2-D colloidal suspension. The beam of an argon ion laser was reflected from a 2-D galvanostatically driven mirror and focused onto the sample plane. The mirror was controlled by a computer in order to scan a quadratic box in the central region of the interference pattern. The scanning frequency was in the order of $50 \mathrm{~Hz}$, therefore a quasi-static optical 
trap with a depth of approximately $30 \mathrm{kT}$ along the contour of the box was obtained. Accordingly, the particles ordered in a 1-D dense packing along the contour of the laser trap and thus served-due to their electrostatic interaction-as a corral for the particles inside the boundary box. Slow variation of the box size thus yields a convenient way to precisely adjust the particle density inside the box as this is essential to these experiments [24].

To investigate the effect of substrate strength on the structure of the colloidal system, spatially resolved averaged particle densities $\rho(x, y)$ were measured by means of video microscopy in the central region of the interference pattern for different laser intensities. The results are shown in Fig. $3 a-d$.

Because the particle density was approximately $5 \%$ below the density required for spontaneous crystallization, the system is in a liquid state when the laser field is turned off (Fig. 3a). When the laser field is adjusted to a value $V_{0}=6 k_{B} T$ the particle distribution is considerably affected and in some regions trimers were already localized at the laser lattice sites defined by the interference pattern (Fig. 3b). Due to thermal fluctuations, however, most of the particles can still diffuse between adjacent laser potential wells. Accordingly, no positional order is observed at these laser intensities. Increasing the light intensity to a value of $V_{0}=17 k_{B} T$, the trimers become more localized at the corresponding triangular lattice sites of the underlying laser field, the latter being indicated schematically as open circles in Fig. 3c. Due to the interplay of the repulsive interparticle forces and the laser potential, now an ordered phase with both longrange positional and orientational order is obtained. In contrast to Fig. 3b, where interwell-diffusion is very pronounced, here, only point defects were observed which can diffuse through the lattice without destroying long-range positional order.

When the laser potential is increased to $V_{0}=$ $27 k_{B} T$, the trimers are even stronger localized to the triagonal laser lattice (Fig. 3d). In contrast to Fig. 3c, however, long-range orientational order becomes partially destroyed again. This is essentially due to the fact, that the lateral extend of the trimers in Fig. $3 d$ is - due to the higher laser intensity - somewhat smaller than in Fig. 3c. Accordingly, the effective coupling between adjacent trimers is weakened and orientational order is partially destroyed. As a consequence, single trimers fluctuate independently in angular direction within the potential wells, which leads to a donut-like structure in Fig. 3d.

It should be noted that the scenario observed here, strongly depends on the relative strengths of the particle-pair interaction vs. their interaction with the light lattice. If, e.g., the Debye screening length would
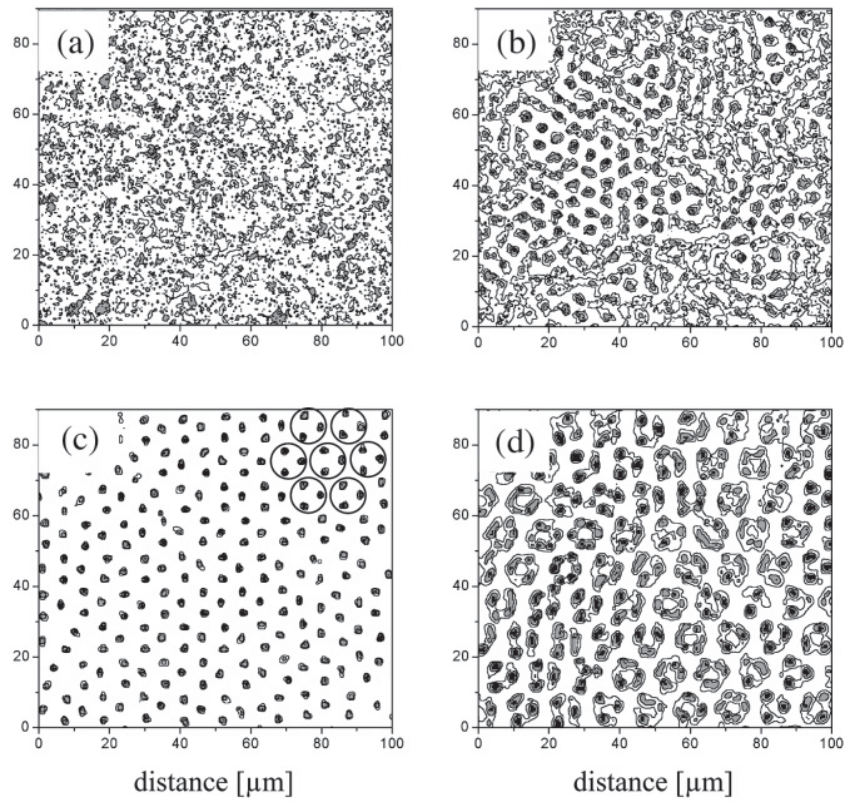

Fig. 3. Density plots of a 2-D colloidal system in the presence of a triangular light lattice at different potential strength $V_{0}$. With increasing $V_{0}$, both positional and orientational order of the trimers is increased until a crystal is obtained (a)-(c). Further increasing to $V_{0}$ leads to loss of orientational order $(D)$.

be much smaller, no orientational coupling between the trimers at intermediate laser fields should be observed.

\section{Single-file diffusion of colloids in one-dimensional channels}

In addition to the phase behavior, lateral confinements have also strong effects on the dynamical properties of colloidal suspensions. This becomes most obvious when colloids diffuse in narrow channels where the mutual passage of particles is impossible. In such a situation which is commonly referred to as single-file diffusion (SFD) the particle self-diffusion is no longer described by a normal diffusion process, but the mean square displacement (MSD) increases as $t^{1 / 2}$ (see, e.g. Kärger and Ruthaven [25]). Besides theoretical interest in this non-Fickian diffusion process SFD, e.g. across membranes, is a crucial intermediate step in many biological and chemical engineering processes such as drug release, particle separation and catalysis.

In order to test the validity of SFD in colloidal systems, Wei et al. confined super paramagnetic particles of $3.6 \mu \mathrm{m}$ diameter to circular trenches fabricated by photolithography [26]. The pair interaction potential of the particles was controlled by an external magnetic field $\mathrm{B}$, which was applied perpendicular 

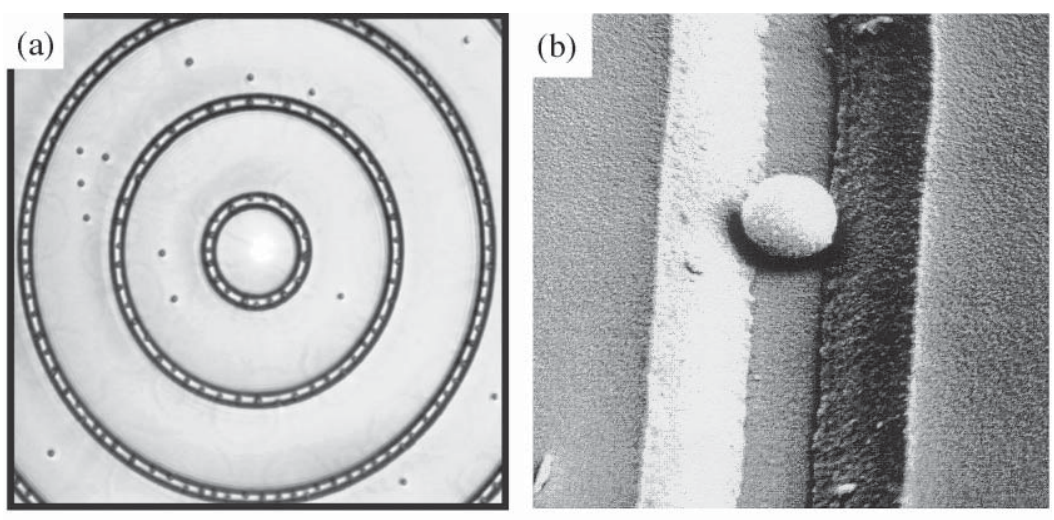

$50 \mu \mathrm{m}$

Fig. 4. Optical micrograph of the topographical structure with narrow channels to establish SFD conditions in a colloidal system (a). Electron microscope picture of the channel structure with a single colloidal particle (b).

to the sample plane and induced a magnetic dipole moment $M$ in the colloids. This leads to a dipole-dipole interaction potential amongst the particles. The bottom plate of the 2-D sample cell was patterned by means of photolithography with a set of concentric circular channels as seen in Fig. 4. The channels are sufficiently narrow to exclude particle passages and the wall interaction can be considered hard spherewall like to a good approximation.

Fig. 5 shows MSDs in a double logarithmic plot for five different magnetic fields, i.e. different pair interaction strengths with the solid lines corresponding to a $t^{1 / 2}$-fit. The data clearly show the expected SFD behavior over more than two decades of time [26]. The small deviations from the solid lines at small time scales correspond to the crossover between the longtime diffusion and the short-time diffusion of the particles. As indicated by the arrows in Fig. 2, the cross-over time from the short-time behavior to SFD is significantly shifted to larger values when the magnetic dipole repulsion between the particles decreases. In addition to the SFD behavior, those measurements confirm for the first time the theoretically predicted Gaussian distribution of particle displacements [27].

Because of the flexibility and ease of varying the channel geometry and the interaction potential form of colloids, the system allows for systematical experimental investigations of many other interesting aspects of SFD, such as the effect of finite channel length [28] and hydrodynamic interactions [29]. The success of one-dimensional colloidal systems in demonstrating the non-Fickian behavior of SFD opens a new avenue not only for the SF systems, but also for particle transport through disordered micro porous mediums, e.g. self-similar percolating pores.

\section{Conclusions}

Colloidal particles have been recognized as excellent model systems for the investigation of various problems in statistical physics such as, e.g. phase transitions. In addition, colloids are also useful for the investigation of non-bulk systems where geometrical constraints such as patterned walls, pores and channels dominate the static and dynamical properties. Because such geometric confinements play an important role in many physical, biological and chemical processes, studies with colloidal suspensions may help

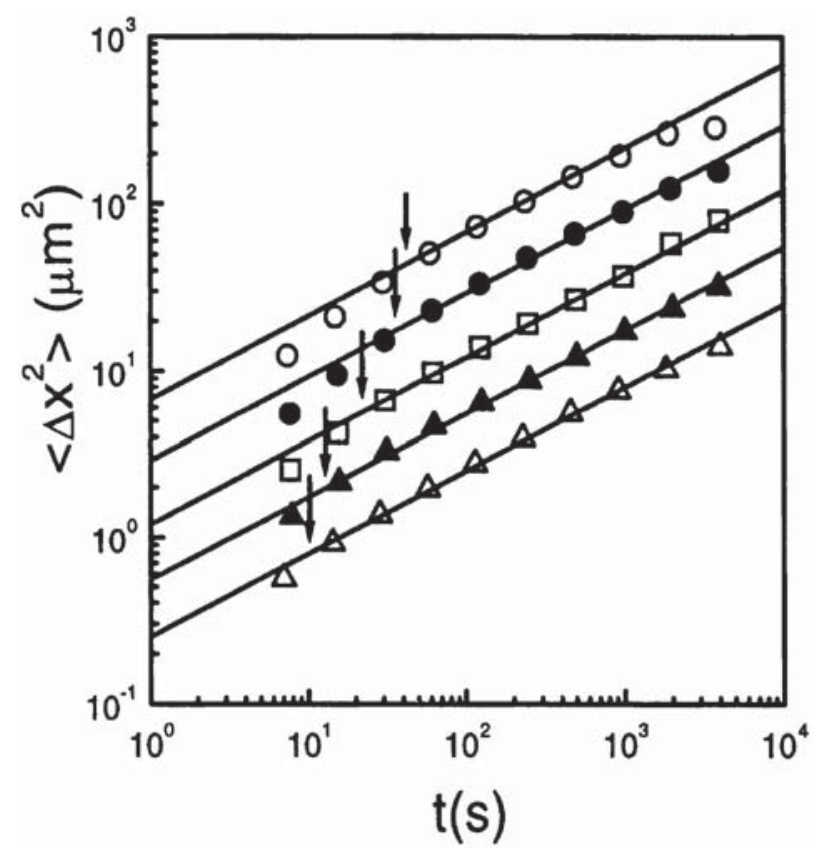

Fig. 5. Particle mean square displacement of super paramagnetic particles in a ring-shaped channel for different magnetic fields vs. time $t$. The solid lines correspond to the predicted $t^{1 / 2}$-behavior [26]. 
to obtain a better understanding of, e.g. transport of particles through cell membranes or catalytic reactions in zeolitic materials.

\section{Acknowledgements}

The author thanks Arjun Yodh and Hartmut Löwen for providing the Figs. 2 and 3 and M. Brunner for helpful discussions.

\section{References}

[1] Gasser U, Weeks ER, Schofield A, Pusey PN, Weitz DA. Real-space imaging of nucleation and growth in colloidal crystallization. Science 2001;292:258-262.

[2] Pertsinidis A, Ling XS. Equilibrium configurations and energetics of point defects in two-dimensional colloidal crystals. Phys Rev Lett 2001;87:098303.

[3] Pertsinidis A, Ling XS. Diffusion of point defects in two-dimensional colloidaly crystals. Nature 2001;413:147-150.

[4] Zahn K, Maret G. Dyamic criteria for melting in two dimensions. Phys Rev Lett 2000;85:3656-3659.

[5] Weeks ER, Crocker JC, Levitt AC, Schofield A, Weitz DA. Three-dimensional direct imaging of structural relaxation near the colloidal glass transition. Science 2000;287:627-630.

[6] Schätzel K, Ackerson BJ. Crystallization of hard sphere colloids. Phys Scripta 1993;T49:70-73.

[7] Pusey PN, Megen Wv. Phase behavior of concentrated suspensions of nearly hard colloidal spheres. Nature 1986; 320:340-342.

[8] Frenkel D, Ladd AJC. New Monte-Carlo method to compute the free-energy of arbitrary solids. Application to the fcc and hcp of hard spheres. J Chem Phys 1984;81:3188-3193.

[9] Tarhan II, Watson GH. Photonic band structure of fcc colloidal crystals. Phys Rev Lett 1996;76:315-318.

[10] Blaaderen Av, Ruel R, Wiltzius P. Template-directed colloidal crystallization. Nature 1997;385:321-323.

[11] Asakura S, Oosawa F. On interaction between two bodies immersed in a solution of macromolecules. J Chem Phys 1954;22:1255.

[12] Götzelmann B, Evans R, Dietrich S. Depletion forces in fluids. Phys Rev E 1998;57:6785.
[13] Mao Y, Cates ME, Lekkerkerker HNW. Depletion force in colloidal systems. Physica A 1995;222:10-24.

[14] Dinsmore AD, Yodh AG. Entropic confinement of colloidal spheres in corners on silicon substrates. Langmuir 1999; 15:314-316.

[15] Roth R, Götzelmann B, Dietrich S. Depletion forces near curved surfaces. Phys Rev Lett 1999;83:448-451.

[16] Lin K-H, Crocker JC, Prasad V, Schofield A, Weitz DA, Lubensky TC, Yodh AG. Entropically driven colloidal crystallization on patterned surfaces. Phys Rev Lett 2000; 85:1770-1773.

[17] Heni M, Löwen H. Surface freezing on patterned substrates. Phys Rev Lett 2000;85:3668-3671.

[18] Heni M, Löwen H. Precrystallization of fluids induced by patterned substrates. J Phys Cond Mater 2001;13:4675-4696.

[19] Ashkin A. Acceleration and trapping of particles by radiation pressure. Phys Rev Lett 1970;24:156-159.

[20] Ashkin A, Dziedzic JM, Bjorkholm JE, Chu S. Observation of a single-beam gradient force optical trap for dielectric particles. Opt Lett 1986;11:288-290.

[21] Loudiyi K, Ackerson BJ. Direct observation of laser induced freezing. Physica A 1992;184:1-25.

[22] Wei Q-H, Bechinger C, Rudhardt D, Leiderer P. Experimental study of laser induced melting in two dimensional colloids. Phys Rev Lett 1998;81:2606-2609.

[23] Brunner M, Bechinger C. Phase behavior of colloidal molecular crystals on trianglular light-lattices. Phys. Rev. Lett. 2002; in press.

[24] Brunner M, Bechinger C, Strepp W, Lobaskin V, Grünberg HHv. Density-dependent pair-interactions in 2D colloidal suspensions. Europhys Lett 2002; in press.

[25] Kärger J, Ruthven DM. Diffusion in zeolites and other microporous solids. New York: Wiley, 1992.

[26] Wei Q-H, Bechinger C, Leiderer P. Single-file-diffusion of colloids in one-dimensional channels. Science 2000; 287:625-627.

[27] Beijeren Hv, Kehr KW, Kutner R. Diffusion in concentrated lattice gases. III. Tracer diffusion on a one-dimensional lattice. Phys Rev B 1983;28:5711-5723.

[28] Hahn K, Kärger J. Deviations from the normal time regime of single-file diffusion. J Phys Chem B 1998;102:5766-5771.

[29] Lin B, Cui B, Lee J-H, Yu J. Hydrodynamic coupling in diffusion of quasi-one-dimensional Brownian particles. Europhys Lett 2002;57:724-730. 